\title{
Pharmacogenetic perspectives on susceptibility to toxic industrial chemicals
}

In the United Kingdom and in the United States the geographical distribution of certain diseases, including some cancers, correlates positively with the degree of industrialisation and hence with the extent of exposure to toxic environmental chemicals. ${ }^{1}$ Nevertheless, attempts to associate specific adverse health effects with exposure to specific toxic chemicals are beset with numerous difficulties. One of the more vexing is that such diseases may not appear until many years after the initial exposure.

A second problem is the extreme diversity of chemical exposure; subjects are often exposed to a broad array of potentially harmful chemicals, rarely just one or two. For many environmental chemicals we do not know the precise sources, prevalence, concentrations, and time course of exposure. With respect to the chemicals themselves, we often remain uncertain of the precise health effects of impurities, contaminants, or environmentally induced alterations in structure. Nor do we know the precise dose of a specific chemical that can produce in each person different pollutant related diseases. Dose response curves for toxicity from environmental chemicals have not been established for different cells of the same tissue, different tissues, different individuals, and different populations. Such information, though presently unobtainable in man, would be most useful in estimating the extent of interindividual variation in susceptibility to toxicity from environmental chemicals.

A further complication is that at different times even the same person becomes more or less susceptible to toxicity from environmental chemicals. Such temporal fluctuations in susceptibility derive from the fact that toxicological sequelae may be greatly influenced by alterations in dynamically interacting host factors. These host factors include age, diet, weight, general health, functional status of various organs, immunological competence, cigarette smoking, ethanol ingestion, and exposure to other xenobiotics. Expression of these host factors may be greatly affected by genetic constitution, behaviour, and environment, all of which also interact in complex, changing ways.
A third problem is the heterogeneity of exposed subjects with respect to critical host factors-for instance, genetic constitution-as well as several other factors enumerated above, that may influence susceptibility. Host factors may play a part not just individually but also through synergistic interactions with each other. Multiple regression analysis is a powerful statistical method that has been used successfully to distinguish the relative contributions of several different causes of variation. In mathematical models thus far applied to pharmacogenetic problems, however, results obtained with regression analysis have been disappointing and conflicting, mainly because standard linear models have been used rather than more complex and appropriate non-linear models tailored individually for each situation. ${ }^{23}$ Results obtained with these previously applied linear models require validation through a prospective, carefully controlled experiment in which only one variable is manipulated independently of others. ${ }^{23}$ Ethical constraints, however, prevent such controlled experiments in man. Therefore, precise causes of large interindividual variations in response to many industrial chemicals remain to be established unequivocally and have had to be approached retrospectively or theoretically or both, through model building exercises.

For these reasons, the identification of the specific causes and host factors responsible for common disorders, such as cancer and heart disease after low, long term exposures to toxic industrial chemicals, is difficult. Numerous causes and host factors may interact in such a way as to obscure the individual role of each factor. Even without exposure to toxic industrial chemicals, several of these factors, such as cigarette smoking or dietary habits, might ultimately have led to the same cancer or cardiopulmonary disorder. By contrast, in Louisville, Kentucky, when hepatic angiosarcoma developed in some workers exposed to vinyl chloride, the extreme rarity of this neoplasm drew attention to the common causal denominator of industrial exposure to a toxic chemical. But inquiry into the cause of this tragedy seems to have stopped 
short of the question of whether workers who developed angiosarcoma did so because of predisposing host factors. This possibility arises because many subjects in this plant were exposed to the same concentrations of vinyl chloride for longer periods without developing angiosarcoma.

In the instance of the Bhopal disaster an industrial accident released high levels of an extremely toxic chemical. The ensuing toxicity unequivocally attributable to methyl isocyanate was not randomly distributed among exposed individuals. Subjects at the extremes of age were most severely affected. Age as a host factor predisposing to toxicity from industrial chemicals is deceptive because it is highly heterogeneous, encompassing many other potentially contributing host factors.

These two examples typify many other retrospective epidemiological studies in that this approach appears to offer the principal way currently available to establish in man a cause of toxicity after exposure to industrial chemicals. Perhaps studies of those who survived without obvious toxic sequelae would illuminate protective host factors.

\section{Pharmacogenetics and ecogenetics: definitions}

Pharmacogenetics and ecogenetics, terms sometimes interchanged, refer to the fields that focus on genetically controlled variations in response to drugs and environmental chemicals, respectively. Ecogenetics is a newly introduced term to focus on genetic variations in response to environmental substances other than drugs; but the methodologies, concepts, and conditions of pharmacogenetics and ecogenetics are generally similar. Whereas numerous examples exis of well characterised genetically determined alter? ations in response to drugs, ${ }^{4-7}$ of which those listec in the table represent only the most intensivelyf investigated, purely ecogenetic entities are fewer an less well defined. Therefore, reviews of ecogenetic? have emphasised pharmacogenetic conditions ancf other entities previously categorised as inborn error\& of metabolism. Since ethical restrictions prohibif experimental administration of toxic chemicals to normal human subjects, the field of ecogenetics has had to be pursued less directly than pharmaco $\overrightarrow{\bar{\omega}}$ genetics.

The present incomplete development of the field op ecogenetics convinced Calabrese that "there is insufficient justification to conduct genetic screenings procedures for the purpose of job denial or transfepo in order to avoid potential occupationally related diseases." 8 He believes, and I concur, that whereas genetic predisposition to occupational chemica 5 toxicity probably exists, present knowledge is to $\sigma^{-}$ fragmentary to justify such actions.

As a result of extreme heterogeneity with respect t\& the numerous contributing host factors listed above $\stackrel{0}{+}$ individuals exhibit large differences in susceptibility to toxicity from environmental chemicals. Pro nounced differences have been observed among sub jects in rates of metabolic activation or inactivation o@ environmental chemicals, of formation and excretion of DNA adducts with these chemicals and theif metabolites, as well as in inherited factors affecting carcinogenicity, immunological capabilities, repaif mechanisms for DNA, antitumour metabolites, an $\overrightarrow{\widehat{B}}$ antioxidants. Thus the critical dose response curve ổ

Monogenic pharmacogenetic conditions and putative aberrant enzymes that affect the metabolism of drugs

\begin{tabular}{|c|c|c|}
\hline Condition* & Aberrant enzyme and normal location & Frequency of affected subjects \\
\hline Acatalasia & Catalase in erythrocytes & $\begin{array}{l}\text { Described in Japan and Switzerland; virtually } \\
\text { absent in UK and USA }\end{array}$ \\
\hline Slow inactivation of isoniazid & $N$-acetyl transferase in liver & $\sim 50 \%$ of white population \\
\hline $\begin{array}{l}\text { Suxamethonium sensitivity or atypical } \\
\text { plasma pseudocholinesterase }\end{array}$ & Pseudocholinesterase in plasma & $\sim 1$ in 1500 subjects \\
\hline $\begin{array}{l}\text { Phenytoin toxicity due to deficient } \\
\text { parahydroxylation (autosomal } \\
\text { dominant) }\end{array}$ & $\begin{array}{l}\text { ? Mixed function oxidase in liver that } \\
\text { p-hydroxylates phenytoin }\end{array}$ & Unknown (only 2 families reported) \\
\hline $\begin{array}{l}\text { Bishydroxycoumarin sensitivity } \\
\text { (mode of inheritance unknown) }\end{array}$ & $\begin{array}{l}\text { ? Mixed function oxidase in liver that } \\
\text { hydroxylates bishydroxycoumarin }\end{array}$ & Unknown (only 1 family reported) \\
\hline $\begin{array}{l}\text { Acetophenetidin induced } \\
\text { methemoglobinaemia }\end{array}$ & $\begin{array}{l}\text { ? Mixed function oxidase in liver that } \\
\text { de-ethylates acetophenetidin }\end{array}$ & $\begin{array}{l}\text { Unknown (possibly this represents an early } \\
\text { description by Shahidi of debrisoquine } \\
\text { polymorphism) }\end{array}$ \\
\hline $\begin{array}{l}\text { Polymorphic serum aryl esterase activity } \\
\text { Deficient } N \text {-hydroxylation of } \\
\text { amobarbital }\end{array}$ & $\begin{array}{l}\text { Serum aryl esterase (paraoxonase) } \\
\text { ? Mixed function oxidase in liver that } \\
N \text {-hydroxylates amobarbital }\end{array}$ & $\begin{array}{l}50 \% \text { of white population } \\
\text { Unknown (only } 1 \text { family reported) }\end{array}$ \\
\hline $\begin{array}{l}\text { Polymorphic hydroxylation of } \\
\text { debrisoquine in man }\end{array}$ & $\begin{array}{l}\text { Mixed function oxidase in liver that } \\
\text { 4-hydroxylates debrisoquine }\end{array}$ & $6-10 \%$ of white population \\
\hline $\begin{array}{l}\text { Polymorphic hydroxylation of } \\
\text { mephenytoin }\end{array}$ & $\begin{array}{l}\text { Mixed function oxidase in liver that } \\
\text { p-hydroxylates } S \text {-mephenytoin }\end{array}$ & $2-5 \%$ of white population \\
\hline \multicolumn{3}{|c|}{ *Autosomal recessive transmission, unless otherwise noted. } \\
\hline
\end{tabular}


toxicity from an environmental chemical may vary significantly from one person to another, as it may even at the same time in the same person from one cell to another or one tissue to another. Regulatory legislation has recognised this broad spectrum of human susceptibility and the existence of multiple special subgroups of particularly sensitive subjects. In setting standards for environmental chemicals the Environmental Protection Agency and the National Institute for Occupational Safety and Health in the United States attempted to protect the most sensitive individuals. For example, under section 109 of the 1983 Clean Air Act that sets standards for photochemical oxidants (ozone), attention was focused on the 3-5\% of the population with asthma, bronchitis, or emphysema. The aim was to protect $99 \%$ of this subset of the total population. As Omenn states:

"A similar process generated an air quality standard for lead which was directed at protecting $99.5 \%$ of exposed children. It is a matter of judgment whether $99.5 \%, 99 \%, 95 \%$, or some other percentage of a population subgroup should be protected, assuming that the standard is enforced and appropriate. These examples serve to illustrate that other important health effects besides carcinogenicity share the problems of extrapolation to low doses and of individual variation in susceptibility, making determination of a noeffect threshold impossible. Unfortunately, the research agenda usually has not addressed this variable of differential susceptibility in any detail." 9

\section{Fundamental principles of pharmacogenetics}

So rapid has progress been in pharmacogenetic research that even full length books on this subject $^{1011}$ can adequately cover only a few of the many developments. Therefore, the scope of this editorial must be limited to a few fundamental principles that pertain to toxicity from industrial chemicals. Several pharmacogenetic principles include complex relations between genetic differences in rates of drug metabolism among subjects and formation of potentially toxic metabolites of a parent drug. In the past the hepatic cytochrome P-450 dependent drug metabolising enzymes were regarded as a "detoxification" system because they convert lipid soluble chemicals that could otherwise remain in the body indefinitely to more polar metabolites readily and rapidly excreted in urine. More recently, however, it has been recognised that this enzyme system may produce potentially toxic, highly reactive metabolites that combine with tissue macromolecules, including DNA, to cause necrosis, immunological reactivity, and mutations.
Qualitative and quantitative differences among subjects in pathways of drug metabolism are believed to be engaged in the regulation and control of such tissue damage. Thus genetic differences among subjects in the enzymes that regulate these pathways render some subjects particularly susceptible to toxicity from different reactive metabolites. Recent work has shown multiple molecular forms or isozymes of cytochrome P-450. Although the precise number of these genetically distinct forms has not been established, thus far 20 such isozymes have been isolated and characterised. These exceedingly heterogeneous isozymes exhibit broad, overlapping substrate specificity and are induced by different chemicals. ${ }^{12}$

Twin and family studies showed genetic control of large differences among normal subjects in the drug metabolising capacity of these proteins. ${ }^{26}$ The extent of this variation ranges from fourfold to 40-fold, depending on the subjects and drugs selected for investigation. A specific example of the toxicological consequences of genetic differences in drug metabolising capacity is the 40 -fold range among normal subjects in the capacity to metabolise chemical carcinogens by a specific cytochrome P-450 dependent isozyme, aryl hydrocarbon hydroxylase (AHH). ${ }^{1314}$ High activities are associated with lung carcinomas but not with familial susceptibility to childhood leukaemia. ${ }^{1314}$ Genetic control of large differences in inducibility of AHH activity have been shown. ${ }^{13-16}$ Presumably, raised AHH activity would produce higher concentrations of toxic reactive intermediates on exposure to polycyclic aromatic hydrocarbons. Reactive intermediates increase chances for covalent binding to DNA, thereby initiating a sequence of events that requires many years to unfold and eventually manifests as a neoplasm.

Another pharmacogenetic condition with implications for susceptibility to toxic industrial chemicals is the polymorphism of the hepatic enzyme $\mathbf{N}$ acetyltransferase. Individuals autosomally recessive for the gene that controls this enzyme conjugate several drugs much more slowly than rapid acetylators. Drugs acetylated by this enzyme include isoniazid, phenelzine, hydralazine, sulphapyridine, and procainamide. Given the same dosing regimen, subjects with the slow phenotype have higher concentrations of these drugs in their bodies than subjects with the rapid phenotype. Therefore, slow acetylators would tend to be more susceptible than rapid acetylators to toxicity from chronic administration of these drugs due to drug accumulation. For isoniazid, such toxicity is manifested as polyneuritis from pyridoxine deficiency; isoniazid competes with pyridoxine for cellular uptake and utilisation.

Subjects with the slow acetylator phenotype were hypothesised to have an increased risk of bladder can- 
cer induced by aromatic amines, since such subjects would have a reduced capacity to detoxify benzidine and arylamines through conjugation. ${ }^{17}$ Arylamines require metabolic activation to initiate carcinogenesis. ${ }^{18}$ Such activation occurs through a hepatic cytochrome P-450 isozyme that hydroxylates the arylamine. In slow acetylators more arylamine would be expected to accumulate secondary to reduced rates of acetylation and hence become available for hydroxylation. Once formed, the hydroxylamine is acetylated to form $\mathrm{N}$-hydroxylamine- $\mathrm{N}$-glucuronide; it is speculated that this metabolite decomposes in the bladder to a highly reactive arylnitrenium ion that combines with nucleic acid to initiate carcinogenesis. ${ }^{18}$ This illustrates the important principle that a genetically controlled block in a metabolic pathway can cause a shift to an alternative, parallel pathway that yields a more toxic final product.

In an urban group of Danish patients with urinary bladder cancer Lower et al reported results that confirmed their hypothesis: slow acetylators were at a 1.74 higher risk of bladder tumours than fast acetylators. ${ }^{17}$ Other studies reviewed by Weber and Hein, however, yielded conflicting results. ${ }^{18}$ Nevertheless, in a dramatic report of bladder tumours arising in workers from Huddersfield, West Yorkshire, chronically exposed to 2-naphthylamine and benzidine, Cartwright et al provided support for the hypothesis of increased risk of bladder tumour in slow acetylators. ${ }^{19}$ Of 23 patients with bladder tumours, 22 were slow acetylators. ${ }^{1920}$ Furthermore, as the rate of acetylation declined among these 22 slow acetylators the invasiveness of the bladder tumours increased. ${ }^{1920}$

The final example to be discussed concerns the human leukocyte antigens (HLA) and their association with increased and decreased risk of certain immunological diseases as well as drug toxicity. The HLA genetic determinants on a single chromosome constitute a haplotype. Every person has two haplotypes, one inherited from each parent. At least four closely linked loci, only a few centimorgans apart on the short arm of human chromosome 6 , control the synthesis of antigens on the surface of leukocytes. Because of their close linkage, the specific genes at these four loci only rarely separate through crossover between homologous chromosomes during meiosis.

Of interest is the extreme genetic heterogeneity of these four loci, more than 100 alleles having been identified, theoretically enabling more than 1000000 different phenotypes. HLA loci and loci closely linked to them function in immune surveillance, protecting the body from several types of environmental assault. For example, these genes serve to combat viral and other pathogens and in the rejection response to foreign tissues. ${ }^{2122}$ Pertinent to this discussion, certain
HLA haplotypes considerably increase susceptibilit to disease associated with immunological impair? ment, initiated in some cases by environmental chem icals. These conditions include chronic active hepa $\stackrel{\rho}{\rightarrow}$ titis, glomerulonephritis, ankylosing spondylitis Reiter's syndrome, rheumatoid arthritis, and dissem inated lupus erythematosus. In certain HLA hap $\frac{\bar{\rho}}{\mathcal{C}}$ lotypes the risk of these disorders, all characterised b\$ a derangement of normal immunological meche anisms, can increase by 40 -fold above that of the population at large. ${ }^{21} 22$

For reasons as yet unclear, some uncommon alleles at the HLA loci may be much more strongly associe ated than other alleles with specific types of response्ల to drugs. For example, in schizophrenic patientș HLA-A1 has been reported to be highly associated with a favourable response to chlorpromazine. ${ }^{23}{ }^{24}{ }_{0}$ By contrast, HLA-A2 appeared to be associated witk an unfavourable response. In patients with affective disorders treated with lithium, HLA-A3 was associ응 ated with a high rate of relapse. ${ }^{25}$ Associations between genetic loci do not necessarily mean that the loci are situated on the same chromosome as the ternfe linkage indicates.

With respect to adverse reactions to drugs, in patients with rheumatoid arthritis treated with sodium aurothiomalate adverse reactions, particu:larly proteinuria, occurred 32 times more frequentlyo in the HLA-DR3 haplotype. ${ }^{26}$ Also in patients with rheumatoid arthritis agranulocytosis after levamisole was encountered much more frequently in subject with the rare haplotype HLA-B27; in a report of three such patients tested eacli possessed the B27 antigen. ${ }^{2}$ Hydralazine induced systemic lupus erythematosus occurred two to three times more often in hyper tensive patients with HLA-DR4 haplotype who? received the drug. ${ }^{28}$ Probably certain HLA haploo types increase susceptibility to toxicity from indus $\overline{\mathbb{}}$ trial chemicals whereas other haplotypes mayo be associated with resistance to such toxicity.

These examples collectively illustrate progress in the rapidly expanding multidisciplinary fields of pharmacogenetics and ecogenetics. The future shouldo disclose many more such conditions, particularly with the application of molecular approaches, including restriction fragment length polymorphisms. Even now, enough examples exist to show that toxicity to industrial chemicals develops not randomly or capri ${ }^{\circ}$ ciously but rather due to diverse, often interacting host factors, including genetically controlled susceptibility.

Department of Pharmacology, E S VESEL I듀

The Pennsylvania State University, College of Medicine, Hershey, PA 17033, USA 


\section{References}

1 Baxter PJ, McDowall ME. Occupation and cancer in London: an investigation into nasal and bladder cancer using the Cancer Atlas. Br J Ind Med 1986;43:44-9.

2 Vesell ES. Selection of subjects for investigation of host factors affecting drug response: a method to identify new pharmacogenetic conditions. Clin Pharmacol Ther 1984;35:1-11.

3 Vesell ES, Penno MB. Assessment of methods to identify sources of interindividual pharmacokinetic variations. Clin Pharmacokinet 1983;8:378-409.

4 Kalow W. Pharmacogenetics: heredity and the response to drugs. Philadelphia: Saunders, 1962.

5 Kalow D. Pharmacoanthropology: drug metabolism. Fed Proc 1984;43:2326-31.

6 Vesell ES. Advances in pharmacogenetics. In: Progress in medical genetics IX. New York and London: Grune and Stratton, 1973:291-367.

7 La Du BN. Pharmacogenetics: defective enzymes in relation to reactions to drugs. Ann Rev Med 1972;23:453-68.

8 Calabrese EJ. Ecogenetics: genetic variation in susceptibility to environmental agents. New York: John Wiley \& Sons, 1984.

9 Omenn GS. Environmental risk assessment: relation to mutagenesis, teratogenesis, and reproductive effects. Journal of American College of Toxicology 1983;2:113-23.

10 Omenn GS, Gelboin HV (eds). Genetic variability in responses to chemical exposure. (Banbury report 16.) New York: Cold Spring Harbor Laboratory, 1984.

11 Kalow W, Goedde HW, Agarwal DP, eds. Ethnic differences in reactions to drugs and xenobiotics. In: Progress in clinical and biological research. Vol 214. New York: Alan R Liss, Inc, 1986.

12 Conney AH. Induction of microsomal cytochrome P-450 enzymes. Life Sci 1986;39:2493-518.

13 Kouri RE, McKinney CE, Slomiany DJ, Snodgrass DR, Wray NP, McLemore TL. Positive correlation between high aryl hydrocarbon hydroxylase activity and primary lung cancer as analyzed in cryopreserved lymphocytes. Cancer Res 1982;42:5030-7.

14 Kouri RE, Levine AS, Edwards BK, McLemore TL, Vesell ES, Nebert DW. Source of interindividual variations in aryl hydrocarbon hydroxylase in mitogen-activated human lymphocytes. In: Omenn GS, Gelboin HV, eds. Genetic variability in responses to chemical exposure. (Banbury report 16.) New York: Cold Spring Harbor Laboratory, 1984.

15 Atlas SA, Vesell ES, Nebert DW. Genetic control of interindividual variations in the inducibility of aryl hydrocarbon hydroxylase in cultured human lymphocytes. Cancer Res 1976;36:4619-30.

16 Okuda T, Vesell ES, Plotkin E, Tarone R, Bast RC, Gelboin HV. Interindividual and intraindividual variations in aryl hydrocarbon hydroxylase in monocytes from monozygotic and dizygotic twins. Cancer Res 1977;37:3904-11.

17 Lower GM, Nilsson T, Nelson CE, Wolf H, Gamsky TE, Bryan GT. N-acetyltransferase phenotype and risk in urinary bladder cancer. Approaches in molecular epidemiology. Preliminary results in Sweden and Denmark. Environ Health Perspect 1979;29:71-9.

18 Weber WW, Hein DW. N-acetylation pharmacogenetics. Pharmacol Rev 1985;37:25-79.

19 Cartwright RA, Glashen RW, Rogers HJ, et al. Role of $\mathrm{N}$-acetyltransferase phenotypes in bladder carcinogenesis: a pharmacogenetic epidemiological approach to bladder cancer. Lancet 1982;ii:842-6.

20 Cartwright RA. Epidemiological studies on $\mathrm{N}$-acetylation and C-center ring oxidation in neoplasia. In: Omenn GS, Gelboin HV, eds. Genetic variability in responses to chemical exposure. (Banbury report 16.) New York: Cold Spring Harbor Laboratory, 1984:359-68.

21 Svejgaard A, Hauge M, Jersild C, et al. The HLA system: an introductory survey. In: Beckman L, Hauge M, Hauge Umea, et al, eds. Monographs in human genetics. Vol 7. Basel: S Karger AG, 1975.

22 Bodmer WF. Inheritance of susceptibility to cancer in man. Oxford: Oxford University Press, 1983.

23 Smeraldi E, Bellodi L, Saccheti E, et al. The HLA system and the clinical response to treatment with chlorpromazine. $\mathrm{Br} J$ Psychiatry 1976;129:486-9.

24 Smeraldi E, Scorza-Smeraldi R. Interference between anti-HLA antibodies and chlorpromazine. Nature 1976;260:532-3.

25 Perris C, Strandman E, Wahlby L. HLA antigens and the response to prophylactic lithium. Neuropsychobiology 1979;5:114-8.

26 Wooley PH, Griffin J, Panayi GS, et al. HLA-DR antigens and toxic reaction to sodium aurothiomalate and D-penicillamine in patients with rheumatoid arthritis. $N$ Engl $J$ Med 1980;303:300-2.

27 Schmidt KL, Mueller-Eckhardt C. Agranulocytosis, levamisole, and HLA-B27. Lancet 1977;ii:85.

28 Batchelor JR, Welsh KI, Mansilla Tinoco R, et al. Hydralazineinduced systemic lupus erythematosus: influence of HLA-DR and sex on susceptibility. Lancet 1980;i:1107-9. 